\title{
LncRNA SNHG1 promotes cell progression and metastasis via sponging miR-377-3p in hepatocellular carcinoma
}

\author{
A. QU ${ }^{1, *}$, Q. YANG ${ }^{2}$ \\ ${ }^{1}$ Department of Radiotherapy, Yidu Central Hospital of Weifang, Weifang, Shandong, China; ${ }^{2}$ Department of Infectious Diseases, Yidu Central \\ Hospital of Weifang, Weifang, Shandong, China \\ ${ }^{*}$ Correspondence: zyttce@163.com
}

Received July 5, 2019 / Accepted September 18, 2019

\begin{abstract}
Hepatocellular carcinoma (HCC) is one of the most famous fatal malignancies in the world. LncRNA SNHG1 has been shown to play roles in the development and progression of various tumors, including HCC. The present study aims to investigate the deeper molecular mechanisms of SNHG1 in HCC. The expression levels of SNHG1 and miR-377-3p were detected by qRT-PCR in HCC tissues and cells. MTT assay was used to examine cell proliferation. Cell apoptosis was evaluated by detecting the apoptotic rate and the protein level of C-caspase 3 using flow cytometry and western blot assays. The protein levels of EMT-related proteins (E-cadherin, N-cadherin, and Vimentin) were measured by western blot. Cell migration and invasion were examined by transwell assay. Xenograft analysis was performed to explore the tumor growth in vivo. The binding sites of SNHG1 and miR-377-3p were predicted by the online software and confirmed by dual-luciferase reporter assay, RNA immunoprecipitation (RIP) assay, and RNA pull-down assay. We found that SNHG1 was markedly upregulated in HCC tissues and cells. Knockdown of SNHG1 induced apoptosis and inhibited proliferation, migration, invasion, and epithelial-to-mesenchymal transition (EMT) of HCC cells. SNHG1 knockdown suppressed the tumor growth of HCC in vivo. SNHG1 directly bound to miR-377-3p. Knockdown of miR-377-3p attenuated the effect of SNHG1 knockdown on proliferation, apoptosis, migration, invasion, and EMT of HCC cells. In conclusion, SNHG1 inhibited apoptosis and induced proliferation, migration, invasion, and EMT by sponging miR-377-3p in HCC, which indicated that SNHG1 may be a potential biomarker and therapeutic target for HCC treatment.
\end{abstract}

Key words: SNHG1, progression, metastasis, miR-377-3p, hepatocellular carcinoma

Hepatocellular carcinoma (HCC) is the most common primary liver cancer, accounting for approximately $75-85 \%$ of cases $[1,2]$. The mortality rate of hepatocellular carcinoma is very high, and the prognosis of HCC has been unsatisfactory over the past few decades [3]. The 5-year overall survival rate of patients with HCC is less than $30 \%$ [4]. However, the molecular mechanisms involved in the initiation and progression of HCC remain poorly understood. Long non-coding RNAs (lncRNAs) are a class of non-coding RNAs (ncRNAs) consisting of more than 200 nucleotides. By exploiting the mechanism of lncRNAs, lncRNAs have been discovered as potential biomarkers and therapeutic targets for tumorigenesis and development [5]. Small nucleolar RNA host gene 1 (SNHG1) is a novel carcinogenic factor that is abnormally expressed in various diseases including colorectal cancer [6], prostate cancer [7], and gastric cancer [8]. In previous research, lncRNA SNHG1 promoted cell proliferation and invasion in liver cancer by binding DNMT1 to inhibit the expression of p53 [9]. In osteosarcoma (OS), the expression of SNHG1 was significantly upregulated, and SNHG1 induced cell proliferation, migration and invasion by regulating miRNA-101-3p considered as a tumor suppressor to elevate the expression of Rho-associated coiled-coil-containing protein kinase 1 (ROCK1) [10]. In addition, there are some reports on the effect of SNHG1 in HCC $[11,12]$. SNHG1 facilitated HCC cell proliferation and blocked cell apoptosis via inhibiting p53 and p53-target genes expression [13]. However, the mechanism of SNHG1 in HCC still needs further research.

MicroRNAs (miRNAs) are short non-coding RNAs composed of 18-25 nucleotides. In recent years, miRNAs have been defined as vital regulators of gene expression by the silencing of complementary target mRNA [14]. The previous study has revealed that miR-377-3p acts as a tumor suppressor and is abnormally expressed in many tumors, such as ovarian cancer [15], non-small cell lung cancer [16] 
and glioma [17]. Accumulating evidence has revealed that lncRNAs function as competing endogenous RNAs (ceRNAs) for miRNAs [18]. We predicted that SNHG1 might interact with miR-377-3p through an online database. Therefore, we speculated that SNHG1 might act as a ceRNA of miR-377-3p. Nevertheless, the role of miR-377-3p in the progression of HCC and the association of SNHG1 with miR-377-3p has not been reported.

In this study, the expression levels of SNHG1 and miR-377-3p in HCC tissues and cells were measured. In addition, the molecular mechanism of SNHG1 in HCC was systematically studied. In conclusion, this study may provide novel therapeutic targets for HCC treatment.

\section{Patients and methods}

Tissue samples. All 35 pairs of HCC tissues and adjacent normal tissues were obtained from patients who underwent surgery at Yidu Central Hospital of Weifang. None of the patients did receive any chemotherapy or radiotherapy before surgery. The research was approved by the Ethics Committee of Yidu Central Hospital of Weifang. Written informed consent was signed by all participants. All tissues were immediately frozen in liquid nitrogen and then stored at $-80^{\circ} \mathrm{C}$.

Cell culture. Human normal hepatocyte cell line (THLE-2) was purchased from American Type Culture Collection (ATCC, Manassas, VA, USA). Human HCC cell lines (Huh-7 and HCCLM3) were obtained from China Center for Type Culture Collection (CCTCC, Wuhan, China). These cells were cultured in Dulbecco's Modified Eagle Medium (DMEM; Gibco, Carlsbad, CA, USA) supplemented with $10 \%$ fetal bovine serum (FBS; Gibco) at $37^{\circ} \mathrm{C}$ with $5 \% \mathrm{CO}_{2}$.

Plasmids and cell transfection. Small interfering RNA (siRNA) targeting SNHG1 (si-SNHG1) and scrambled control (Scramble), pcDNA-SNHG1 (SNHG1) and the control pcDNA (Vector) were purchased from RiboBio (Guangzhou, China). The control mimic (NC) and miR-377-3p mimic (miR-377-3p), miR-377-3p inhibitor (anti-miR-377-3p) and the negative control (anti-NC) were synthesized by HanBio Technology (Shanghai, China). Cell transfection was performed using Lipofectamine 2000 (Invitrogen, Carlsbad, CA, USA) following the manufacturer's instructions. Lentiviruses containing shRNA against SNHG1 (sh-SNHG1) or negative control (sh-NC) were obtained from GenePharma (Shanghai, China). For lentiviral infection, cells $\left(2 \times 10^{5}\right.$ cells/well) were incubated in $1 \times 10^{6} \mathrm{TU} / \mathrm{ml}$ viral suspension supplemented with polybrene. Stably transfected cells were selected using puromycin.

Quantitative real-time PCR. Total RNA was extracted from tissues or cells using Trizol reagent (Invitrogen) as described by the manufacturer. Then, RNA reverse transcription was performed by High-Capacity cDNA Reverse Transcription Kits (Thermo Fisher Scientific, Waltham,
MA, USA). The expression levels were detected using SYBR Green Mixture (Takara, Dalian, China). GAPDH was used as an internal control. Primers used were as follows: SNHG1-F (forward, 5'-GGAGCGAGATCCCTCCAAAAT-3'), SNHG1-R (reverse, 5'-GGCTGTTGTCATACTTCTCATGG-3'), miR-377-3p-F (forward, 5'-GAGCAGAGGTTGCCCTTG-3'), miR-377-3p-R (reverse, 5'-ACAAAAGTTGCCTTTGTGTGA-3'), GAPDH-F (forward, 5'-GACTCCACTCACGGCAAATTCA-3'), GAPDH-R (reverse, 5'-TCGCTCCTGGAAGATGGTGAT-3').

MTT assay. $5 \times 10^{3}$ cells were seeded in 96-well plates after transfection. Then, MTT was added to each well after incubation for $24 \mathrm{~h}, 48 \mathrm{~h}$ and $72 \mathrm{~h}$, and then incubated for 3 $\mathrm{h}$ at $37^{\circ} \mathrm{C}$. Intracellular formazan crystals were dissolved by dimethyl sulfoxide (DMSO; Sigma, St Louis, MO, USA). The absorbance was measured at $490 \mathrm{~nm}$ by a microplate reader (Bio-Rad, Hercules, CA, USA).

Flow cytometry. The transfected cells were resuspended and stained with FITC Annexin V Apoptosis Detection Kit II (BD Biosciences, Franklin Lakes, NJ, USA) according to the protocols of the manufacturer. The cell apoptotic rate was assessed by flow cytometry (BD Biosciences) following the manufacturer's instructions.

Western blot assay. Total protein was lysed by RIPA lysis buffer (Thermo Fisher Scientific). Proteins were quantified using the BCA Protein Assay Kit (Pierce, Appleton, WI, USA) following the manufacturer's protocol. Then, proteins were separated by SDS-PAGE gel electrophoresis and transferred to polyvinylidene fluoride membranes (Millipore, Billerica, MA, USA). Next, the membranes were blocked by $5 \%$ skim milk (Nestlé, Shuangcheng, China) for $2 \mathrm{~h}$ at $37^{\circ} \mathrm{C}$ and then incubated overnight at $4{ }^{\circ} \mathrm{C}$ with primary antibodies against C-caspase 3 (ab13847, 1:2,000; Abcam, Cambridge, UK), pro-caspase 3 (ab32150, 1:2,000; Abcam), E-cadherin (ab15148, 1:2,000; Abcam), Vimentin (ab137321, 1:2,000; Abcam), N-Cadherin (ab18203, 1:2,000; Abcam), and GAPDH (ab9485, 1:2,000; Abcam). Subsequently, the membranes were washed three times with TBST and incubated with horseradish peroxidase (HRP)-conjugated secondary antibody (ab205718, 1:4,000; Abcam) for $2 \mathrm{~h}$ at $37^{\circ} \mathrm{C}$. Finally, the protein bands were visualized by the enhanced chemiluminescence system (Thermo Fisher Scientific) and quantitated using ImageJ software (National Institutes of Health, Bethesda, MD, USA). GAPDH was used as an endogenous control.

Transwell assay. For cell migration analysis, the transfected cells were seeded in serum-free medium and plated into the upper chamber of a 24-well transwell with $8 \mu \mathrm{m}$ polycarbonate membrane filters (Thermo Fisher Scientific). The medium of the lower chamber contained $10 \%$ fetal bovine serum (FBS). Cells were incubated for $24 \mathrm{~h}$ with 5\% $\mathrm{CO}_{2}$ at $37^{\circ} \mathrm{C}$, the cells adhered to the lower surface were fixed by paraformaldehyde and stained with crystal violet. For invasion analysis, the upper layer of the polycarbonate membrane was coated with $100 \mu \mathrm{l}$ of diluted Matrigel (BD 
A

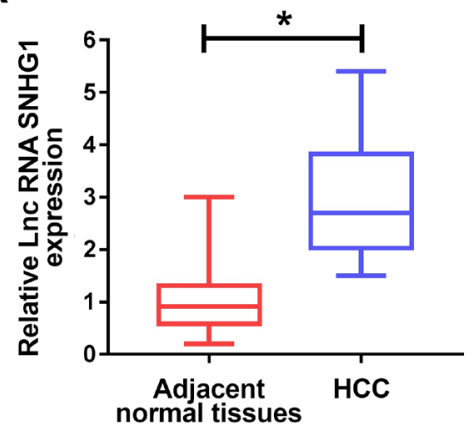

B

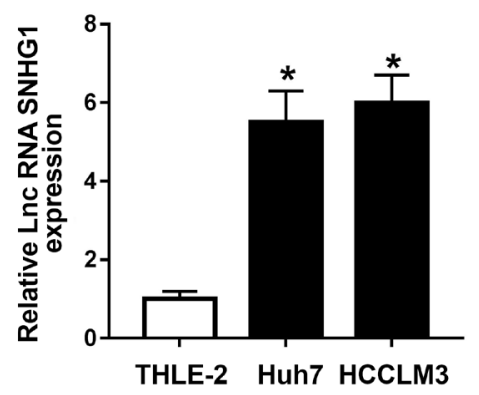

Figure 1. LncRNA SNHG1 was upregulated in HCC tissues and cell lines. A) qRT-PCR assay was performed to examine the expression of SNHG1 in HCC tissues and adjacent normal tissues. B) The expression of SNHG1 was detected in a normal human liver cell line (THLE-2) and HCC cell lines (Huh-7 and HCCLM3). ${ }^{*} \mathrm{p}<0.05$.

Biosciences) and the rest of the methods were the same as for detecting cell migration.

Xenograft mice experiment. Male BALB/c nude mice (6 weeks) were maintained in clean conditions and used for xenograft assay. Huh-7 and HCCLM3 cells were transfected with lentivirus harboring sh-SNHG1 or sh-NC, respectively. Subsequently, cells were subcutaneously injected into nude mice. Tumor volume was measured every 5 days and tumor weight was detected after mice were sacrificed. The xenograft mice experiment was approved by the Committee on Animal Welfare of Yidu Central Hospital of Weifang.

Dual-luciferase reporter assay. The binding sites of SNHG1 and miR-377-3p were predicted by the online software starBase v2.0. The SNHG1-wt or SNHG1-mut sequences containing the miR-377-3p wild or mutant binding sites were cloned into pGL3 plasmids (Promega, Madison, WI, USA). The constructed pGL3 vectors were then co-transfected into Huh-7 and HCCLM3 cells with miR-377-3p mimic (miR-377-3p) or the control mimic (NC) using Lipofectamine 2000 (Invitrogen). Luciferase activity was detected with the Dual-Luciferase Reporter Assay System (Promega) according to the manufacturer's instructions. The activity of Renilla luciferase was used as a standardized reference.

RNA Immunoprecipitation (RIP) assay. Magna RIP Kit (Millipore) was used to perform the RIP assay according to the manufacturer's instructions. Briefly, Huh-7 and HCCLM3 cells transfected with the control mimic (NC) or miR-377-3p mimic (miR-377-3p) were collected and resuspended in RIP lysis buffer with protease inhibitor and RNase inhibitor. Then, cell lysates were incubated with RIP buffer containing magnetic beads conjugated Anti-Ago2 (Millipore) for $2 \mathrm{~h}$ at $4^{\circ} \mathrm{C}$. Finally, the co-precipitated RNAs were detected by qRT-PCR.

Pull-down assay. Biotin-labeled miR-377-3p probe (Bio-miR-377-3p) and the corresponding control probe (Bio-NC) were purchased from RiboBio. In brief, the biotinylated probe was incubated with M-280 Streptavidin
Dynabeads (Invitrogen) at $37^{\circ} \mathrm{C}$ for $2 \mathrm{~h}$ to form probecoated beads. Then Huh-7 and HCCLM3 cells were lysed and incubated with probe-coated beads at $4^{\circ} \mathrm{C}$ for $3 \mathrm{~h}$. Finally, the enrichment of SNHG1 was examined by qRT-PCR.

Statistical analysis. All data were displayed as mean \pm standard deviation with three independent experiments. The difference was checked by Student's t-test and one-way analysis of variance (ANOVA). Statistical analysis was implemented using GraphPad Prism 7 software (GraphPad, San Diego, CA, USA). A p-value $<0.05$ was taken as statistically significant.

\section{Results}

LncRNA SNHG1 was upregulated in HCC tissues and cell lines. To explore the expression of SNHG1 in HCC tissues and cells, we measured SNHG1 expression using qRT-PCR. The results revealed that SNHG1 expression was dramatically upregulated in HCC tissues compared with adjacent normal tissues (Figure 1A). Likewise, SNHG1 expression was strikingly higher in HCC cell lines (Huh-7 and HCCLM3) than normal human liver cell line (THLE-2, Figure 1B). These results suggested that SNHG1 may be associated with hepatocellular carcinoma.

Knockdown of SNHG1 inhibited cell proliferation and induced apoptosis in HCC cells. In vitro, Huh-7 and HCCLM3 cells were transfected with Scramble or si-SNHG1 to investigate the effects of SNHG1 in HCC. The result exhibited that knockdown of SNHG1 significantly decreased the expression level of SNHG1 in both Huh-7 and HCCLM3 cells compared to the negative control (Figure 2A). To analyze the effects of SNHG1 on HCC progression, we measured cell proliferation and apoptosis using MTT and flow cytometry assays. These results showed that transfection of si-SNHG1 remarkably restrained cell proliferation (Figure 2B), and markedly facilitated apoptosis rates in Huh-7 and HCCLM3 cells (Figure 2C). Furthermore, western blot revealed that the protein level of C-caspase 3 was higher in HCC cells trans- 
A

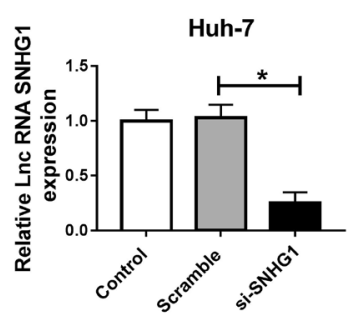

C

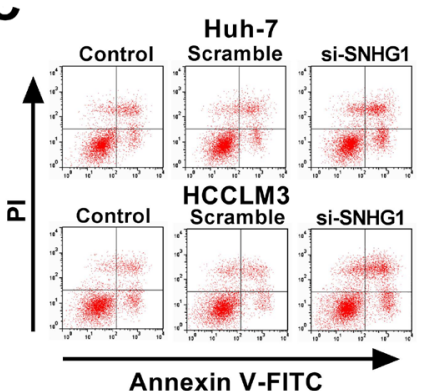

E

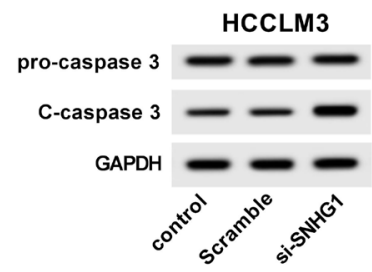

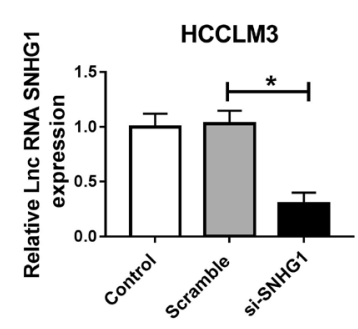

B

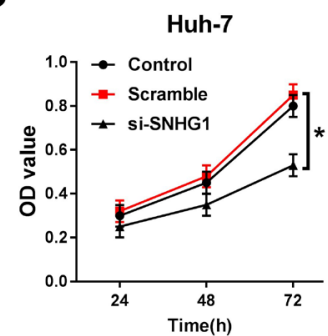

D
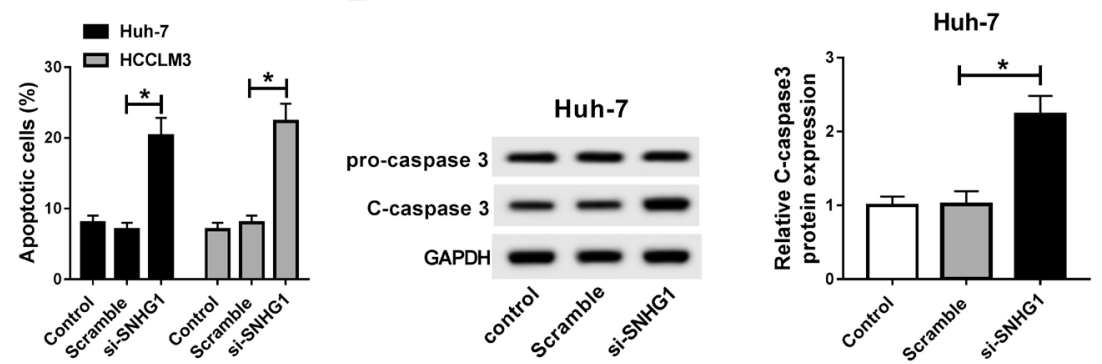

Figure 2. Knockdown of SNHG1 inhibited cell proliferation and induced apoptosis in HCC cells. A-D) Huh-7 and HCCLM3 cells were transfected with Scramble or si-SNHG1. A) SNHG1 expression was measured by qRT-PCR in transfected cells. B) Cell proliferation was detected by MTT assay at 24 $\mathrm{h}, 48 \mathrm{~h}$ and $72 \mathrm{~h}$ after transfection. C) Cell apoptotic rate was evaluated using flow cytometry at $48 \mathrm{~h}$ upon transfection. D and E) The protein level of C-caspase 3 was measured by western blot. ${ }^{*} \mathrm{p}<0.05$.

fected with si-SNHG1 than that in cells transfected with Scramble (Figures 2D, 2E). These data suggested that SNHG1 knockdown inhibited cell proliferation and promoted apoptosis in HCC cells.

Knockdown of SNHG1 suppressed cell migration, invasion, and EMT in HCC cells. Firstly, transwell analysis indicated that transfection of si-SNHG1 resulted in a notable increase in cell migration (Figure 3A) and invasion (Figure 3B) in Huh-7 and HCCLM3 cells. To investigate the effect of SNHG1 on EMT, we examined EMT-related protein levels by western blot. The results showed that knockdown of SNHG1 significantly increased the protein level of E-cadherin, and obviously decreased the protein levels of $\mathrm{N}$-cadherin and Vimentin in Huh-7 and HCCLM3 cells (Figure 3C). Taken together, these results demonstrated that SNHG1 inhibited cell migration, invasion, and EMT in HCC cells.

Knockdown of SNHG1 blocked the tumor growth in vivo. To evaluate the potential effect of SNHG1 on HCC tumorigenesis in vivo, a mouse xenograft model was established. Huh-7 and HCCLM3 cells transfected with sh-NC or sh-SNHG1 were subcutaneously injected into nude mice. Compared with the negative control group, tumor volume and weight of the sh-SNHG1 group were prominently decreased (Figures 4A, 4B). Similarly, the results of the injection of HCCLM3 cells were consistent with the above results (Figures 4D, 4E). Moreover, we also determined SNHG1 expression in the tumor tissues by qRT-PCR analysis. SNHG1 expression was obviously lower in the sh-SNHG1 group than that in the sh-NC group (Figures $4 \mathrm{C}, 4 \mathrm{~F}$ ). Thus, these data concluded that SNHG1 knockdown suppressed the tumor growth of HCC in vivo.

SNHG1 acted as a sponger of miR-377-3p. To explore the molecular mechanism of SNHG1 in HCC, we predicted that miR-377-3p and SNHG1 have putative binding sites by online database starBase v2.0 (Figure 5A). Next, a dualluciferase reporter assay was performed to confirm whether 

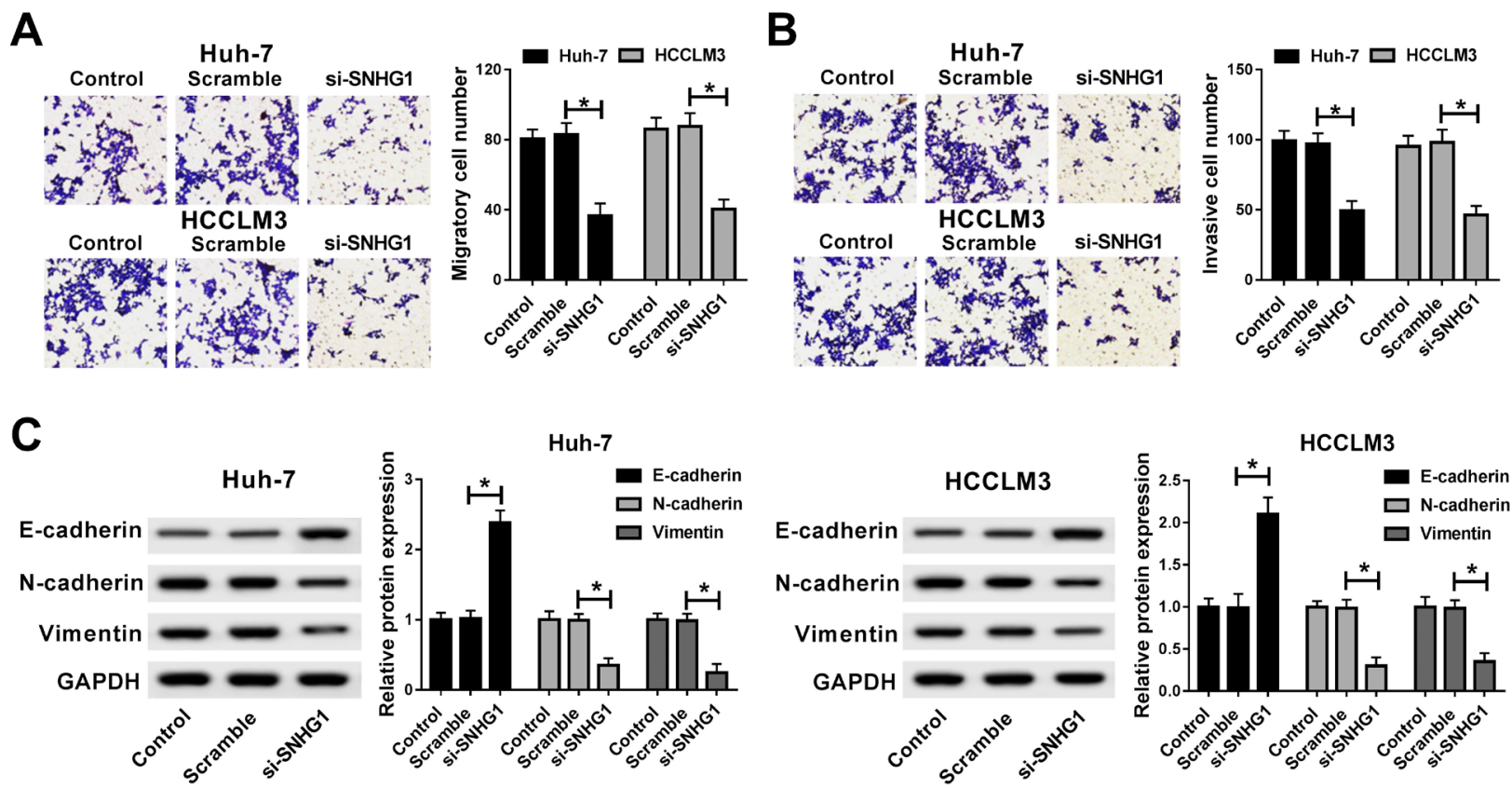

Figure 3. Knockdown of SNHG1 suppressed cell migration, invasion, and EMT in HCC cells. A-C) Huh-7 and HCCLM3 cells were transfected with Scramble or si- SNHG1. A and B) Cell migration and invasion were detected by transwell assay. C) The protein levels of EMT-related proteins (Ecadherin, $\mathrm{N}$-Cadherin, and Vimentin) were examined by western blot assay. ${ }^{\star} \mathrm{p}<0.05$.

A

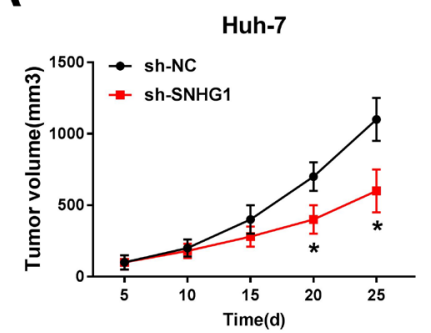

D

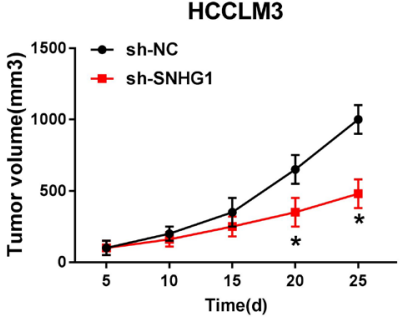

B

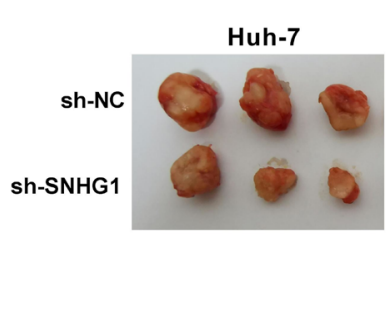

$\mathbf{E}$

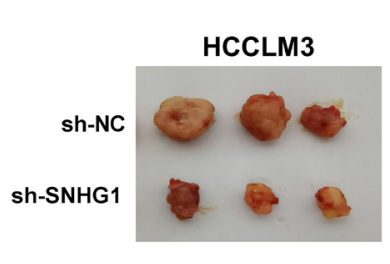

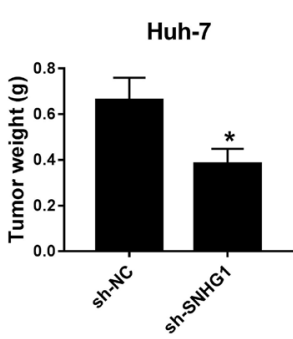

C

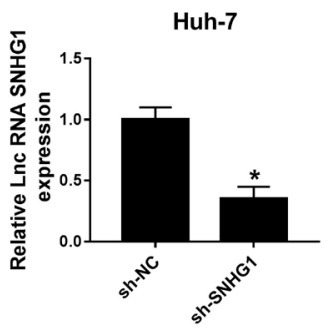

$\mathbf{F}$

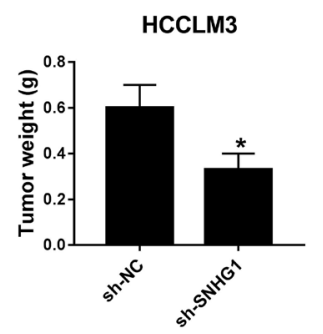

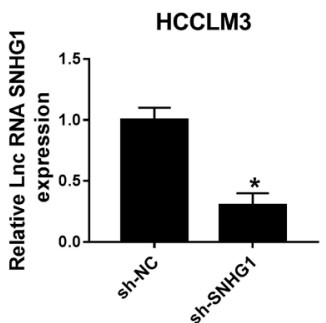

Figure 4. Knockdown of SNHG1 blocked the tumor growth in vivo. A-F) Huh-7 and HCCLM3 cells were transfected with sh-NC or sh-SNHG1 and subcutaneously injected into nude mice. A and D) Tumor volume was measured every 5 days. B and E) Tumor weight was detected after mice were sacrificed. $C$ and F) The expression of SNHG1 was examined by qRT-PCR.

SNHG1 targets miR-377-3p. The results showed that mature miR-377-3p apparently reduced the luciferase activity of the SNHG1-wt group, whereas it had no inhibitory effect on the luciferase activity of the SNHG1-mut group compared to the control in Huh-7 and HCCLM3 cells (Figure 5B). Moreover,
RIP and pull-down assays were performed to further validate the relationship between SNHG1 and miR-377-3p in Huh-7 and HCCLM3 cells. RIP assay showed that SNHG1 was notably enriched in the miR-377-3p group coated with the Ago2 antibody compared with the control group (Figure 5C), 
A

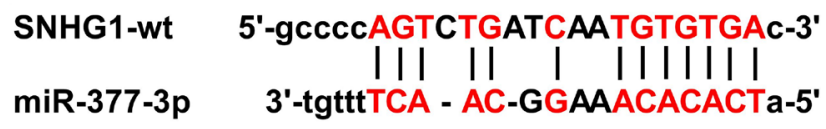

SNHG1-mut 5'-gccccTCACACATGAAACACACTc-3'

B

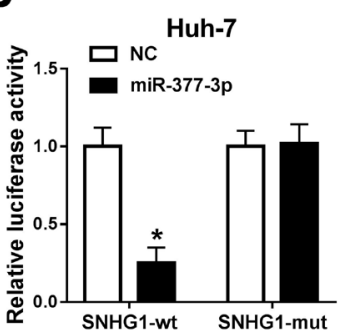

D

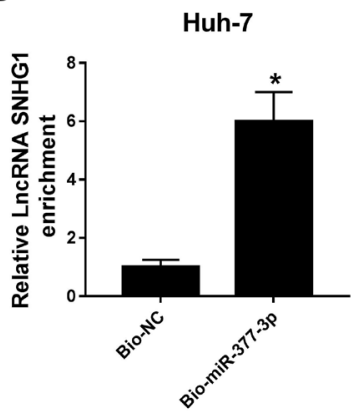

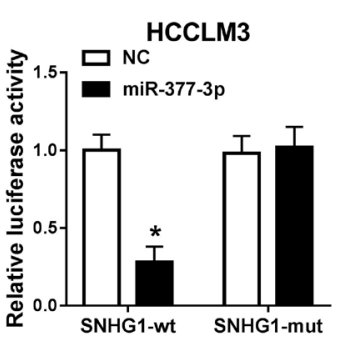

C
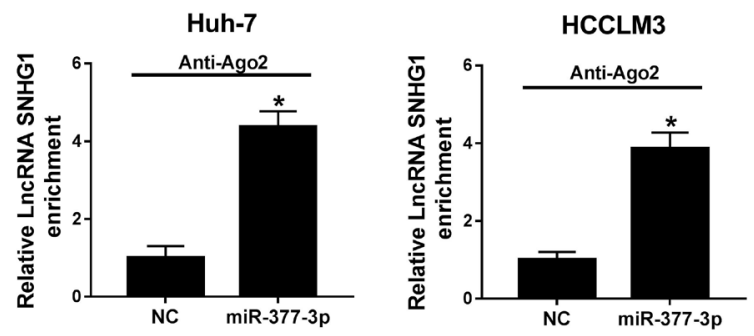

$\mathbf{E}$

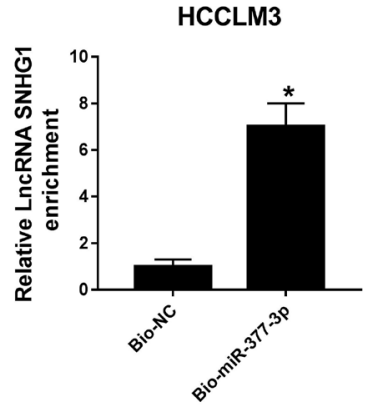

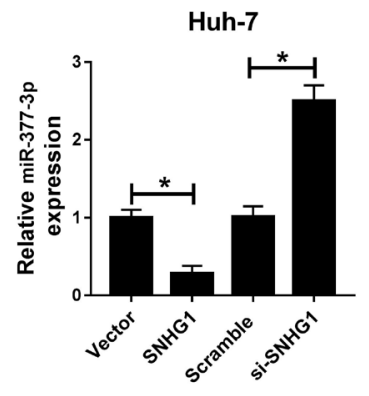

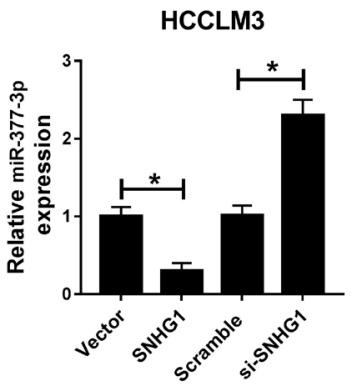

Figure 5. SNHG1 directly targeted miR-377-3p. A) The putative binding sites of SNHG1 and miR-377-3p. B) Huh-7 and HCCLM3 cells were cotransfected with SNHG1-wt or SNHG1-mut luciferase reporter vectors and miR-377-3p mimic (miR-377-3p) or the control mimic (NC), and luciferase activity was examined at $48 \mathrm{~h}$ after transfection. C) Huh-7 and HCCLM3 cells were transfected with miR-377-3p mimic (miR-377-3p) or the control mimic (NC), and RIP assay was performed to determine SNHG1 enrichment in the Ago2 immunoprecipitation complex. D) Huh-7 and HCCLM3 cells were transfected with Bio-miR-377-3p or Bio-NC, and RNA pull-down assay was carried out to detect SNHG1 enrichment. E) Huh-7 and HCCLM3 cells were transfected with pcDNA (Vector), pcDNA-SNHG1 (SNHG1), Scramble, and si-SNHG1, respectively. The expression levels of miR-377-3p were detected by qRT-PCR. * $\mathrm{p}<0.05$.

and pull-down analysis revealed that SNHG1 was significantly enriched by Bio-miR-377-3p, but not by Bio-NC (Figure 5D). These data suggested that SNHG1 might interact with miR-377-3p in HCC.

Next, the expression of miR-377-3p was detected in Huh-7 and HCCLM3 cells transfected with pcDNA (Vector), pcDNA-SNHG1 (SNHG1), Scramble and si-SNHG1, respectively. The results showed that overexpression of SNHG1 strikingly reduced the expression of miR-377-3p, whereas knockdown of SNHG1 markedly upregulated the expression level of miR-377-3p in Huh-7 and HCCLM3 cells (Figure 5E). Taken together, these results demonstrated that SNHG1 regulated miR-377-3p.

Knockdown of miR-377-3p attenuated the effects of the SNHG1 knockdown on proliferation and apoptosis of HCC cells. First, we examined the miR-377-3p expression in HCC tissues and cells by qRT-PCR. The results indicated that miR-377-3p was remarkably downregulated in HCC tissues compared with adjacent normal tissues (Figure 6A), and the expression level of miR-377-3p was dramatically lower in Huh-7 and HCCLM3 cells than THLE-2 cells (Figure 6B). We further explored the relationship of SNHG1 and miR-377-3p in Huh-7 and HCCLM3 cells transfected with Scramble, si-SNHG1, si-SNHG1+anti-NC, and si-SNHG1+anti-miR377-3p, respectively. The results indicated that the expression of miR-377-3p was markedly increased in the si-SNHG1 group, while miR-377-3p was remarkably downregulated after simultaneous inhibition of SNHG1 and miR-377-3p (Figure 6C). Meanwhile, the knockdown of SNHG1 significantly inhibited cell proliferation (Figure 6D) and promoted apoptosis (Figure 6E) of Huh-7 and HCCLM3 cells. In addition, Huh-7 and HCCLM3 cells transfected with si-SNHG1 had a higher protein level of C-caspase 3 than the control group (Figure 6F). However, these results caused by SNHG1 knockdown could be abrogated by inhibition of SNHG1 and miR-377-3p, suggesting that SNHG1 could regulate HCC cell proliferation and apoptosis by mediating miR-377-3p. 

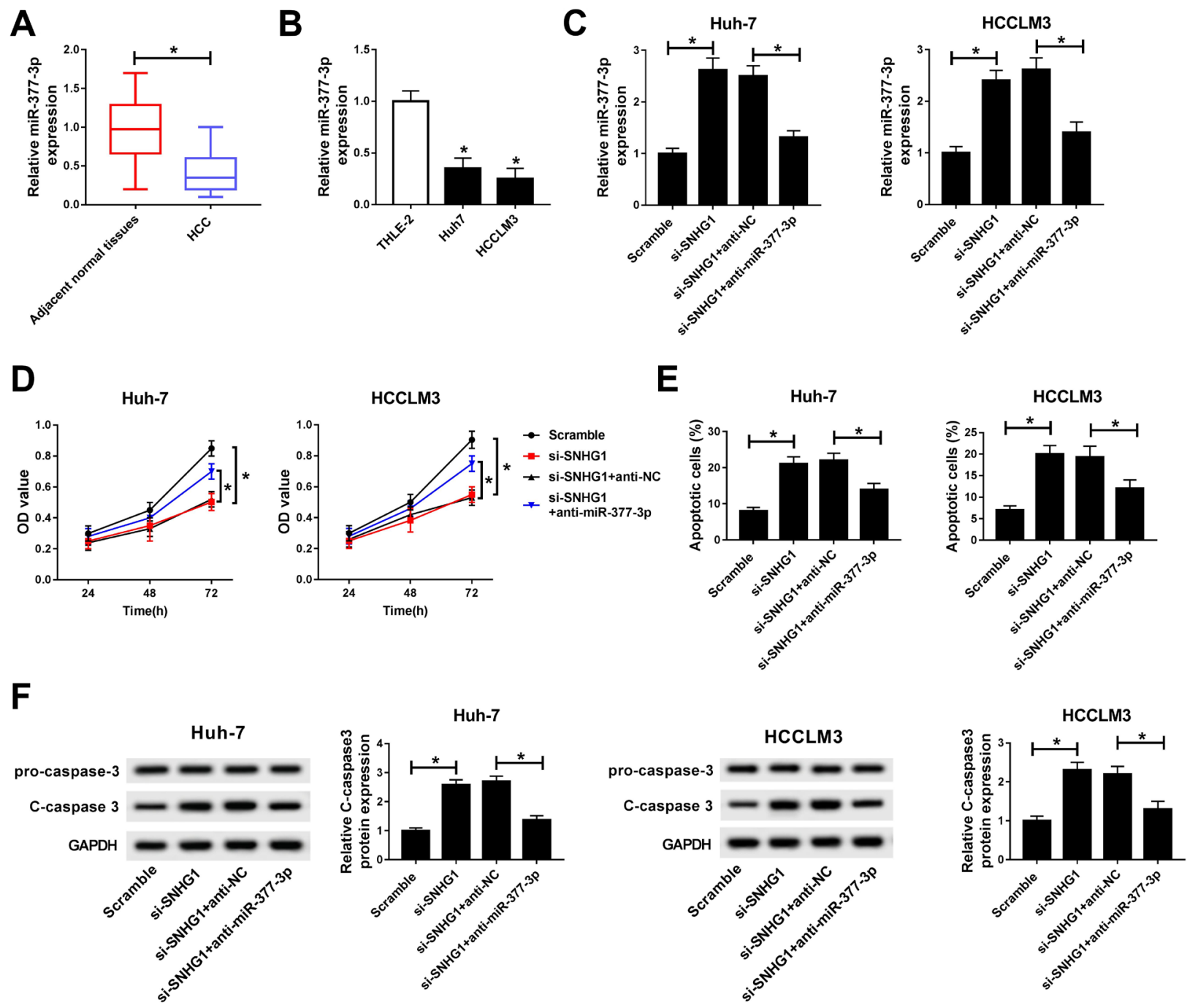

Figure 6. Knockdown of miR-377-3p attenuated the effects of SNHG1 knockdown on proliferation and apoptosis of HCC cells. A) The expression of miR-377-3p was measured by qRT-PCR in HCC tissues and adjacent normal tissues. B) The expression of miR-377-3p was detected in a normal human liver cell line (THLE-2) and HCC cell lines (Huh-7 and HCCLM3). C-F) Huh-7 and HCCLM3 cells were transfected with Scramble, si-SNHG1, si-SNHG1+anti-NC, and si-SNHG1+anti-miR-377-3p, respectively. C) The expression of miR-377-3p was measured by qRT-PCR at $48 \mathrm{~h}$ post-transfection. D) Cell proliferation was evaluated by MTT assay at $24 \mathrm{~h}, 48 \mathrm{~h}$, and $72 \mathrm{~h}$ after transfection. E) Cell apoptotic rate was detected by flow cytometry. F) The protein level of C-caspase 3 was measured by western blot. ${ }^{*} \mathrm{p}<0.05$.

Knockdown of miR-377-3p attenuated the inhibition of SNHG1 knockdown on migration, invasion, and EMT of HCC cells. Furthermore, transwell assay revealed that cell migration and invasion were significantly inhibited after transfecting si-SNHG1 into Huh-7 and HCCLM3 cells, but co-transfection of si-SNHG1 and anti-miR-377-3p abolished the effects (Figures 7A, 7B). In addition, the protein level of E-cadherin was obviously lower, and the protein levels of $\mathrm{N}$-cadherin and Vimentin were apparently higher in the si-SNHG1+anti-miR-377-3p group than that in the si-SNHG1 group (Figure 7C). All these data suggested that the knockdown of miR-377-3p reversed the effects of SNHG1 knockdown on migration, invasion, and EMT of HCC cells.

\section{Discussion}

The prognosis of hepatocellular carcinoma is very poor with a high recurrence rate [4]. In recent years, the studies suggested that lncRNAs dysregulation was associated with the progression of cancers, including HCC $[3,19]$. Therefore, it's meaningful to discover novel therapeutic targets for HCC treatment.

LncRNA SNHG has been identified as an oncogene in various cancers [20, 21]. SNHG1 has been widely reported to be an oncogenic factor in many cancers, such as pancreatic cancer, non-small cell lung cancer, and gastric cancer [22-24]. Increasing evidence suggested that SNHG1 was 
A

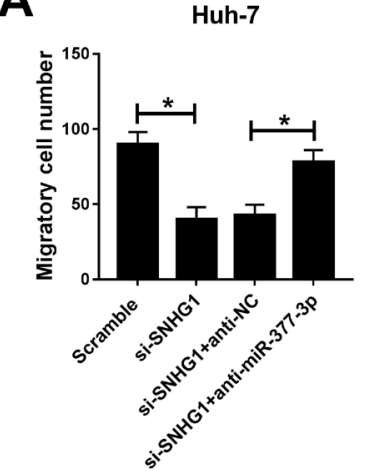

C
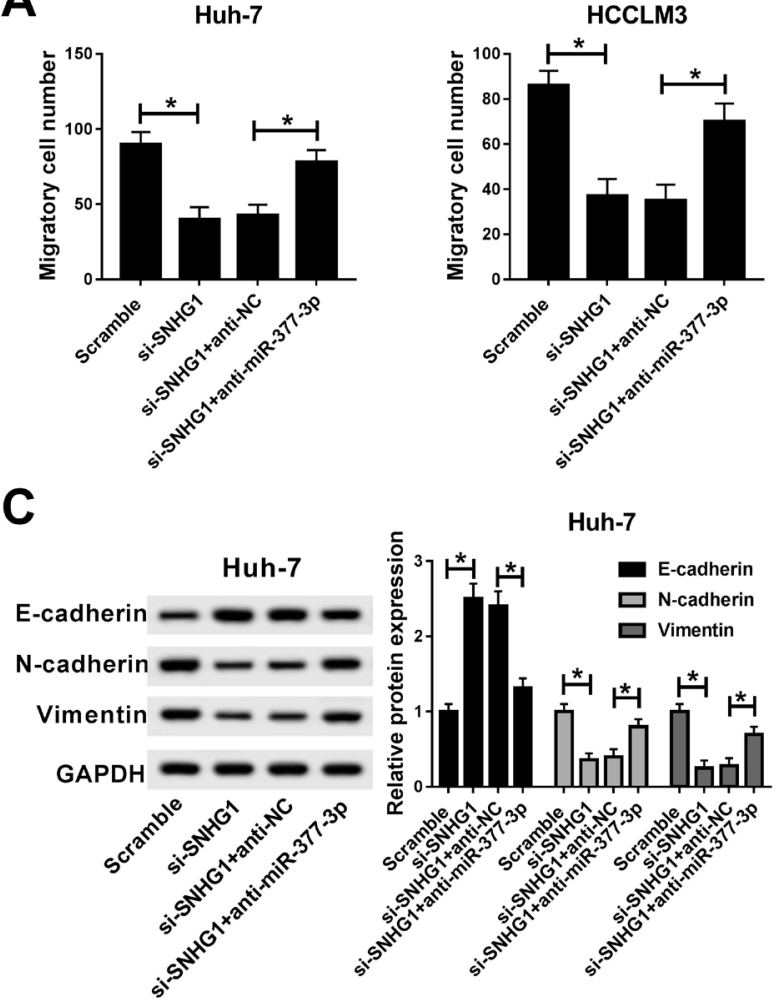

B
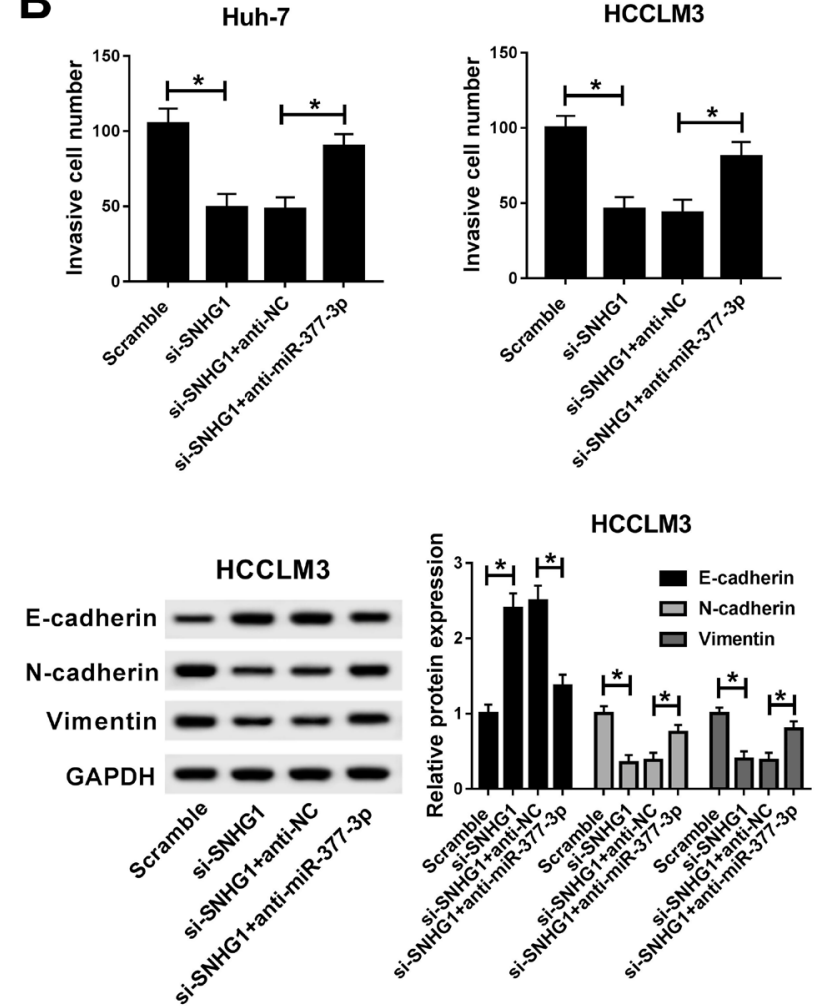

Figure 7. Knockdown of miR-377-3p attenuated the inhibition of SNHG1 knockdown on migration, invasion, and EMT of HCC cells. A-C) Huh-7 and HCCLM3 cells were transfected with Scramble, si-SNHG1, si-SNHG1+anti-NC, and si-SNHG1+anti-miR-377-3p, respectively. A and B) Cell migration and invasion were evaluated by transwell assay after transfection. $\mathrm{C}$ ) The protein levels of EMT-related proteins (E-cadherin, $\mathrm{N}-\mathrm{Cadherin}$, and Vimentin) were detected by western blot. ${ }^{\star} \mathrm{p}<0.05$.

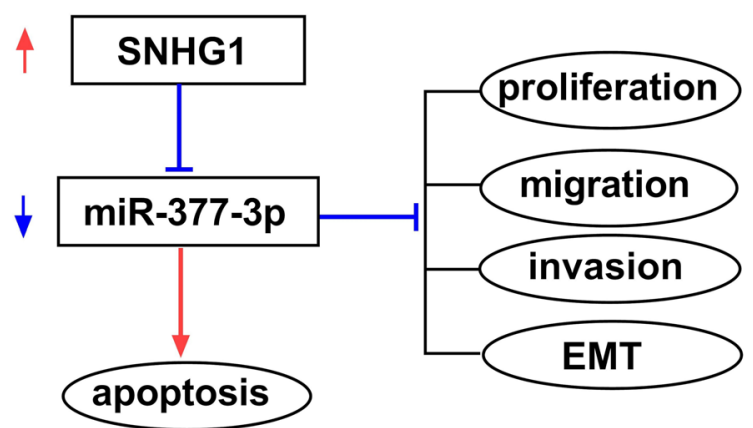

Figure 8. The cell pathway map demonstrates the mechanism of SNHG1 promoting HCC progression. Decreased level of miR-377-3p due to the upregulation of SNHG1 results in diminished inhibition of proliferation, migration, invasion, and EMT as well as the attenuation of apoptosis. Contrarily, knockdown of SNHG1 supports apoptosis and inhibits proliferation, invasion, and EMT through the increased level of miR-377-3p.

associated with metastasis and prognosis in cancer patients $[11,25]$. SNHG1 silencing could ameliorate cisplatin resistance of NSCLC via targeting miR-140-5p [26]. The previous study confirmed that SNHG1 as a competing endogenous RNA (ceRNA) for DNMT1 contributed to cell proliferation and invasion in liver cancer by inhibiting the expression of p53 [9]. In cholangiocarcinoma (CCA), SNHG1 regulated growth and tumorigenesis through elevating the expression of toll-like receptor 4 (TLR4) and stimulating NF- $\kappa B$ signaling by sponging miR-140 [27]. In glioma, SNHG1 regulated the malignant behavior of cells by binding microRNA-154-5p or miR-376b-3p to increase the expression of its downstream target gene FOXP2 [28]. In addition, accumulating evidence indicates that lncRNA SNHG1 functioned as a carcinogenic factor in HCC. Li et al. indicated that the nuclear expression of SNHG1 was enhanced by miR-21 and contributed to sorafenib resistance by activating the Akt pathway in HCC cells [29]. SNHG1 was dramatically upregulated in HCC tissues and cells and accelerated proliferation, invasion, and migration of HCC cells by downregulating the expression of miR-195, suggesting that miR-195 was a direct target of SNHG1 [30]. SNHG1 was a prognostic biomarker and therapeutic target for HCC [13]. Consistent with the previous study, the expression of SNHG1 in HCC tissues and cells was dramatically higher than that in the normal liver tissues and cells, which suggests that SNHG1 may play vital roles in HCC growth and progression.

Moreover, this study indicated that SNHG1 could competitively bind with miR-377-3p. MiR-377-3p has been reported as a tumor inhibitor or tumor promoter in cancers. Liu et 
al. demonstrated that miR-377-3p was significantly upregulated in colorectal cancer (CRC) tissues, promoted cell proliferation and epithelial-mesenchymal transition (EMT), and inhibited apoptosis in CRC cells by activating NF- $\mathrm{KB}$ pathway and upregulating glycogen synthase kinase- $3 \beta$ (GSK-3 $\beta$ ) expression [31]. MiR-377-3p functioned as a tumor inhibitor in many cancers, such as ovarian cancer [15], non-small cell lung cancer [16], and glioma [17]. In hepatocellular carcinoma, miR-377 was downregulated [32-34]. Ge et al. exhibited that miR-377 suppressed cell proliferation and increased apoptosis by targeting Bcl-xL 3'UTR in HCC [32]. Moreover, miR-377 restrained proliferation, migration, and invasion of HCC cells via regulating IRX3 [33]. MiR-377 was prominently downregulated in HCC tissues and cells and blocked HCC cell proliferation and invasion by targeting $\mathrm{T}$ lymphoma invasion and metastasis 1 (TIAM1) [34]. In this study, we further confirmed that miR-377-3p was significantly downregulated in HCC tissues and cells. The study also showed that miR-377-3p was regulated by SNHG1 and abolished the effect of SNHG1 on HCC progression, which suggested that miR-377-3p might be a direct target of SNHG1 in HCC. Besides, we have drawn a simple cell pathway map (Figure 8).

In conclusion, this study suggested that SNHG1 was upregulated in HCC tissues and cells. SNHG1 might promote hepatocellular carcinoma progression and metastasis by sponging miR-377-3p, suggesting that SNHG1 was a promising therapeutic target for HCC treatment.

\section{References}

[1] TIAN M, SHI Y, LIU W, FAN J. Immunotherapy of hepatocellular carcinoma: strategies for combinatorial intervention. Sci China Life Sci 2019; 62: 1138-1143. https://doi. org/10.1007/s11427-018-9446-2

[2] BUONAGURO L, MAURIELlO A, CAVALlUZZO B, PETRIZZO A, TAGLIAMONTE M. Immunotherapy in hepatocellular carcinoma. Ann Hepatol 2019; 18: 291-297. https://doi.org/10.1016/j.aohep.2019.04.003

[3] MAI H, ZHOU B, LIU L, YANG F, CONRAN C et al. Molecular pattern of lncRNAs in hepatocellular carcinoma. J Exp Clin Cancer Res 2019; 38: 198. https://doi.org/10.1186/ s13046-019-1213-0

[4] JIANG X, TAN HY, TENG S, CHAN YT, WANG D et al. The role of amp-activated protein kinase as a potential target of treatment of hepatocellular carcinoma. Cancers (Basel). 2019; 11: E647. https://doi.org/10.3390/cancers11050647

[5] WANG J, ZHANG X, CHEN W, HU X, LI J et al. Regulatory roles of long noncoding RNAs implicated in cancer hallmarks. Int J Cancer 2020; 146: 906-916. https://doi. org/10.1002/ijc.32277

[6] XU M, CHEN X, LIN K, ZENG K, LIU X et al. The long noncoding RNA SNHG1 regulates colorectal cancer cell growth through interactions with EZH2 and miR-154-5p. Mol Cancer 2018; 17: 141. https://doi.org/10.1186/s12943018-0894-x
[7] LI J, ZHANG Z, XIONG L, GUO C, JIANG T et al. lncRNA negatively regulates miR-199a-3p to enhance CDK7 expression and promote cell proliferation in prostate cancer. Biochem Biophys Res Commun 2017; 487: 146-152. https://doi. org/10.1016/j.bbrc.2017.03.169

[8] HU Y, MA Z, HE Y, LIU W, SU Y et al. LncRNA-SNHG1 contributes to gastric cancer cell proliferation by regulating DNMT1. Biochem Biophys Res Commun 2017; 491: 926931. https://doi.org/10.1016/j.bbrc.2017.07.137

[9] LI SJ, WANG L, SUN ZX, SUN SJ, GAO J et al. LncRNA SNHG1 promotes liver cancer development through inhibiting p53 expression via binding to DNMT1. Eur Rev Med Pharmacol Sci 2019; 23: 2768-2776. https://doi. org/10.26355/eurrev_201904_17550

[10] DENG R, ZHANG J, CHEN J. lncRNA SNHG1 negatively regulates miRNA-101-3p to enhance the expression of ROCK1 and promote cell proliferation, migration and invasion in osteosarcoma. Int J Mol Med 2019; 43: 1157-1166. https://doi.org/10.3892/ijmm.2018.4039

[11] TAN C, CAO J, CHEN L, XI X, WANG S et al. Noncoding RNAs Serve as Diagnosis and Prognosis Biomarkers for Hepatocellular Carcinoma. Clin Chem 2019; 65: 905-915. https://doi.org/10.1373/clinchem.2018.301150

[12] ZHU Q, YANG H, CHENG P, HAN Q. Bioinformatic analysis of the prognostic value of the lncRNAs encoding snoRNAs in hepatocellular carcinoma. Biofactors 2019; 45: 244252. https://doi.org/10.1002/biof.1478

[13] ZHANG M, WANG W, LI T, YU X, ZHU Y et al. Long noncoding RNA SNHG1 predicts a poor prognosis and promotes hepatocellular carcinoma tumorigenesis. Biomed Pharmacother 2016; 80: 73-79. https://doi.org/10.1016/j. biopha.2016.02.036

[14] ZUZIC M, ROJO ARIAS JE, WOHL SG, BUSSKAMP V. Retinal miRNA Functions in Health and Disease. Genes (Basel). 2019; 10: E377. https://doi.org/10.3390/genes10050377

[15] TANG L, YANG B, CAO X, LI Q, JIANG L et al. MicroRNA-377-3p inhibits growth and invasion through sponging JAG1 in ovarian cancer. Genes Genomics 2019; 41: 919-926. https://doi.org/10.1007/s13258-019-00822-w

[16] ZHANG J, LI Y, DONG M, WU D. Long non-coding RNA NEAT1 regulates E2F3 expression by competitively binding to miR-377 in non-small cell lung cancer. Oncol Lett 2017; 14: 4983-4988. https://doi.org/10.3892/ol.2017.6769

[17] LIU Y, GAO Y, LI D, HE L, IW L et al. LASP1 promotes glioma cell proliferation and migration and is negatively regulated by miR-377-3p. Biomed Pharmacother 2018; 108: 845-851. https://doi.org/10.1016/j.biopha.2018.09.068

[18] YAMAMURA S, IMAI-SUMIDA M, TANAKA Y, DAHIYA R. Interaction and cross-talk between non-coding RNAs. Cell Mol Life Sci 2018; 75: 467-484. https://doi.org/10.1007/ s00018-017-2626-6

[19] ABBASTABAR M SARFI M, GOLESTANI A, KHALILI E. lncRNA involvement in hepatocellular carcinoma metastasis and prognosis. EXCLI J 2018; 17: 900-913. https://doi. org/10.17179/excli2018-1541 
[20] GAO XF, HE HQ, ZHU XB, XIE SL, CAO Y. LncRNA SNHG20 promotes tumorigenesis and cancer stemness in glioblastoma via activating PI3K/Akt/mTOR signaling pathway. Neoplasma 2019; 66: 532-542. https://doi.org/10.4149/ neo_2018_180829N656

[21] LI W, XU W, SONG JS, WU T, WANG WX. LncRNA SNHG16 promotes cell proliferation through miR-302a-3p/ FGF19 axis in hepatocellular carcinoma. Neoplasma 2019; 66: 397-404. https://doi.org/10.4149/neo_2018_180720N504

[22] CUI L, DONG Y, WANG X, ZHAO X, KONG C et al. Downregulation of long noncoding RNA SNHG1 inhibits cell proliferation, metastasis, and invasion by suppressing the Notch-1 signaling pathway in pancreatic cancer. J Cell Biochem 2019; 120: 6106-6112. https://doi.org/10.1002/ jcb. 27897

[23] LI Z, LU Q, ZHU D, HAN Y, ZHOU X et al. Lnc-SNHG1 may promote the progression of non-small cell lung cancer by acting as a sponge of miR-497. Biochem Biophys Res Commun 2018; 506: 632-640. https://doi.org/10.1016/j. bbrc.2018.10.086

[24] GUO W, HUANG J, LEI P, GUO L, LI X. LncRNA SNHG1 promoted HGC-27 cell growth and migration via the miR140/ADAM10 axis. Int J Biol Macromol 2019; 122: 817-823. https://doi.org/10.1016/j.ijbiomac.2018.10.214

[25] YU J, YAN Y, HUA C, MING L. Upregulation of lncRNA SNHG1 is associated with metastasis and poor prognosis in cancers: A meta-analysis. Medicine (Baltimore) 2019; 98: e15196. https://doi.org/10.1097/MD.0000000000015196

[26] SHI SL, ZHANG ZH. Long non-coding RNA SNHG1 contributes to cisplatin resistance in non-small cell lung cancer by regulating $\mathrm{miR}-140-5 \mathrm{p} / \mathrm{Wnt} / \beta$-catenin pathway. Neoplasma 2019; 66: 756-765. https://doi.org/10.4149/ neo_2018_181218N980

[27] LI Z, LI X, DU X, ZHANG H, WU Z et al. The interaction between lncRNA SNHG1 and miR-140 in regulating growth and tumorigenesis via TLR4/NF- $\mathrm{BB}$ pathway in cholangiocarcinoma. Oncol Res 2019; 27: 663-672. https://doi.org/10. 3727/096504018X15420741307616
[28] LI H, XUE Y, MA J, SHAO L, WANG D et al. SNHG1 promotes malignant biological behaviors of glioma cells via microRNA-154-5p/miR-376b-3p- FOXP2- KDM5B participating positive feedback loop. J Exp Clin Cancer Res 2019; 38: 59. https://doi.org/10.1186/s13046-019-1063-9

[29] LI W, DONG X, HE C, TAN G, LI Z et al. LncRNA SNHG1 contributes to sorafenib resistance by activating the Akt pathway and is positively regulated by miR-21 in hepatocellular carcinoma cells. J Exp Clin Cancer Res 2019; 38: 183. https://doi.org/10.1186/s13046-019-1177-0

[30] ZHANG H, ZHOU D, YING M, CHEN M, CHEN P et al. Expression of Long Non-Coding RNA (lncRNA) Small Nucleolar RNA Host Gene 1 (SNHG1) Exacerbates Hepatocellular Carcinoma Through Suppressing miR-195. Med Sci Monit 2016; 22: 4820-4829. https://doi.org/10.12659/ msm.898574

[31] LIU WY, YANG Z, SUN Q, YANG X, HU Y et al. miR-377$3 p$ drives malignancy characteristics via upregulating GSK$3 \beta$ expression and activating NF- $\kappa \mathrm{B}$ pathway in hCRC cells. J Cell Biochem 2018; 119: 2124-2134. https://doi.org/10.1002/ jcb. 26374

[32] GE H, ZOU D, WANG Y, JIANG H, WANG L. MicroRNA-377 Downregulates bcl-xl and increases apoptosis in hepatocellular carcinoma cells. Oncol Res 2017; 25: 29-34. https://doi.org/10.3727/096504016X14719078133168

[33] WANG P, ZHUANG C, HUANG D, XU K. Downregulation of miR-377 contributes to IRX3 deregulation in hepatocellular carcinoma. Oncol Rep 2016; 36: 247-252. https://doi. org/10.3892/or.2016.4815

[34] CHEN G, LU L, LIU C, SHAN L, YUAN D. MicroRNA-377 suppresses cell proliferation and invasion by inhibiting TIAM1 expression in hepatocellular carcinoma. PLoS One 2015; 10: e0117714. https://doi.org/10.1371/journal. pone. 0117714 